\title{
Tanshinone IIA inhibits angiogenesis in human endothelial progenitor cells in vitro and in vivo
}

\author{
Hsiang-Ping Lee ${ }^{1,2, *}$, Yueh-Ching Liu ${ }^{3, *}$, Po-Chun Chen ${ }^{4}$, Huai-Ching Tai ${ }^{5,6,7}$, Te-Mao \\ $\mathrm{Li}^{1}$, Yi-Chin Fong ${ }^{8,9}$, Chih-Shiang Chang ${ }^{10}$, Min-Huan Wu ${ }^{11,12}$, Li-Pin Chiu ${ }^{13,14}$, Chia- \\ Jung Wang ${ }^{15}$, Yi-Hsuan Chen ${ }^{15}$, Yih-Jer Wu ${ }^{15}$, Chih-Hsin Tang ${ }^{4,16,17}$ and Shih-Wei \\ Wang ${ }^{15,18}$ \\ ${ }^{1}$ Graduate Institute of Chinese Medicine, China Medical University, Taichung, Taiwan \\ ${ }^{2}$ Department of Chinese Medicine, China Medical University Hospital, Taichung, Taiwan \\ ${ }^{3}$ Department of Orthopaedics, Mackay Memorial Hospital, Taipei, Taiwan \\ ${ }^{4}$ Graduate Institute of Biomedical Science, China Medical University, Taichung, Taiwan \\ ${ }^{5}$ School of Medicine, Fu-Jen Catholic University, New Taipei City, Taiwan \\ ${ }^{6}$ Department of Urology, Fu-Jen Catholic University Hospital, New Taipei City, Taiwan \\ ${ }^{7}$ Department of Urology, National Taiwan University Hospital, Taipei, Taiwan \\ ${ }^{8}$ Department of Sports Medicine, College of Health Care, China Medical University, Taichung, Taiwan \\ ${ }^{9}$ Department of Orthopedic Surgery, China Medical University Beigang Hospital, Yun-Lin County, Taiwan \\ ${ }^{10}$ Graduate Institute of Pharmaceutical Chemistry, China Medical University, Taichung, Taiwan \\ ${ }^{11}$ Physical Education Office, Tunghai University, Taichung, Taiwan \\ ${ }^{12}$ Sports Recreation and Health Management Continuing Studies, Tunghai University, Taichung, Taiwan \\ ${ }^{13}$ Department of Nursing, Taipei City Hospital, Taipei, Taiwan \\ ${ }^{14}$ General Education Center, University of Taipei, Taipei, Taiwan \\ ${ }^{15}$ Department of Medicine, Mackay Medical College, New Taipei City, Taiwan \\ ${ }^{16}$ Department of Pharmacology, School of Medicine, China Medical University, Taichung, Taiwan \\ ${ }^{17}$ Department of Biotechnology, College of Health Science, Asia University, Taichung, Taiwan \\ ${ }^{18}$ Graduate Institute of Natural Products, College of Pharmacy, Kaohsiung Medical University, Kaohsiung, Taiwan \\ *These authors have contributed equally to this work \\ Correspondence to: Shih-Wei Wang, email: shihwei@mmc.edu.tw \\ Chih-Hsin Tang, email: chtang@mail.cmu.edu.tw
}

Keywords: endothelial progenitor cells; angiogenesis; tanshinone IIA; VEGF-A

Received: July 26, $2017 \quad$ Accepted: October 30, $2017 \quad$ Published: November 24, 2017

Copyright: Lee et al. This is an open-access article distributed under the terms of the Creative Commons Attribution License 3.0 (CC BY 3.0), which permits unrestricted use, distribution, and reproduction in any medium, provided the original author and source are credited.

\section{ABSTRACT}

Accumulating evidence reports that bone marrow-derived endothelial progenitor cells (EPCs) regulate angiogenesis, postnatal neovascularization and tumor metastasis. It has been suggested that understanding the molecular targets and pharmacological functions of natural products is important for novel drug discovery. Tanshinone IIA is a major diterpene quinone compound isolated from Danshen (Salvia miltiorrhiza) and is widely used in traditional Chinese medicine (TCM). Evidence indicates that tanshinone IIA modulates angiogenic functions in human umbilical vein endothelial cells. However, the anti-angiogenic activity of tanshinone IIA in human EPCs has not been addressed. Here, we report that tanshinone IIA dramatically suppresses vascular endothelial growth factor (VEGF)-promoted migration and tube formation of human 
EPCs, without cytotoxic effects. We also show that tanshinone IIA markedly inhibits VEGF-induced angiogenesis in the chick embryo chorioallantoic membrane (CAM) model. Importantly, tanshinone IIA significantly attenuated microvessel formation and the expression of EPC-specific markers in the in vivo Matrigel plug assay in mice. Further, we found that tanshinone IIA inhibits EPC angiogenesis through the PLC, Akt and JNK signaling pathways. Our report is the first to reveal that tanshinone IIA reduces EPC angiogenesis both in vitro and in vivo. Tanshinone IIA is a promising natural product worthy of further development for the treatment of cancer and other angiogenesis-related pathologies.

\section{INTRODUCTION}

Angiogenesis is a critical step in the physiology of tissue repair, bone remodeling and reproduction, as well as embryonic development [1]. Angiogenesis also plays an important role during pathological processes, including various inflammatory diseases and tumor progression, as well as metastasis [2-4]. Inhibiting angiogenesis is therefore a critical strategy in cancer therapy and other angiogenesis-related disorders $[5,6]$. Currently, more than 10 angiogenesis inhibitors are in clinical use. Most target the vascular endothelial growth factor (VEGF) receptor (VEGFR) axis, using small molecule inhibitors or antibody agents $[7,8]$. Since VEGF and its receptors are critical regulators of angiogenesis in tumor cells, VEGF and VEGFR signaling are promising therapeutic targets in cancer metastasis and treatment.

Bone marrow-derived endothelial progenitor cells (EPCs) contribute to postnatal physiological and pathological neovascularization [9]. EPCs have cellular subpopulations and contain different functional capacities such as migration, proliferation, and recruitment in response to angiogenesis. Circulating EPCs, a cell population characterized by the CD133/CD34/VEGFR2 phenotype, are mobilized from the bone marrow into the bloodstream and contribute to neovascularization by direct incorporation into neovessels [10]. Tumor-secreted growth factors, such as VEGF, control the mobilization of EPCs, which subsequently contribute to cancer growth and angiogenesis of certain tumors [11]. It has been suggested that EPCs act as critical regulators of the angiogenic switch that controls the development of micrometastasis and subsequently promotes tumor macrometastasis [12]. All of this evidence supports the role of EPCs in tumor angiogenesis and metastasis. Selective targeting of EPCs may have potential as an anti-angiogenic strategy against cancer metastasis.

Several natural products have been documented to reduce angiogenesis via different mechanisms [13-15]. Danshen, the dried root of Salvia miltiorrhiza Bunge, is a well-known herb in traditional Chinese medicine (TCM) that is mainly used clinically in preventative or therapeutic preparations for hepatitis, arthritis, stroke, vascular disease, coronary heart disease and cancer [16, 17]. Tanshinone IIA is a major diterpene extracted from danshen that possesses anti-atherosclerotic, anti-inflammatory and anti-oxidative effects [18-20]. Tanshinone IIA inhibits angiogenesis in human umbilical vein endothelial cells (HUVECs) [21, 22], but its anti-angiogenic effects in EPCs are largely unknown. In this study, we report that tanshinone IIA inhibits EPC migration and tube formation without any evidence of cytotoxic activity. Moreover, tanshinone IIA impedes angiogenesis and the expression of progenitor cell markers in vivo. Tanshinone IIA shows potential as a promising angiogenesis inhibitor that targets EPCs.

\section{RESULTS}

\section{Tanshinone IIA shows no cytotoxicity in human EPCs}

We first used the MTT assay to examine the effect of tanshinone IIA upon cell viability in EPCs. Tanshinone IIA $(1-10 \mu \mathrm{M})$ slightly reduced cell growth of EPCs after $24 \mathrm{~h}$ of treatment (Figure 1A). To investigate whether this effect was caused by cytotoxicity, we performed the lactate dehydrogenase (LDH) assay in EPCs. As shown in Figure 1B, treatment with tanshinone IIA for $24 \mathrm{~h}$ did not induce LDH release, even at a high concentration of tanshinone IIA $(30 \mu \mathrm{M})$. In addition, prolonged treatment of tanshinone IIA for $48 \mathrm{~h}$ did not induce LDH release in EPCs (Supplementary Figure 1). Therefore, we suggest that tanshinone IIA exhibits safe activity without causing cytotoxicity in human EPCs.

\section{Tanshinone IIA inhibits VEGF-induced migration and tube formation of EPCs}

VEGF is the most crucial angiogenic factor during physiological and pathological angiogenesis. Migration of EPCs through normal tissue boundaries (the basement membrane) is an important event in neovessel synthesis [30]. We used Transwell chambers to investigate the effects of tanshinone IIA on EPC migration. We found that tanshinone IIA inhibited VEGF-promoted migration in a concentration-dependent manner (Figure 2A). We next performed the tube formation assay to validate the anti-angiogenic activity of tanshinone IIA in EPCs. As shown in Figure 2B, VEGF stimulation resulted in EPC reorganization and formation of capillary-like structures. Tanshinone IIA significantly suppressed VEGF-induced tube formation of EPCs. Collectively, these results indicate 
A

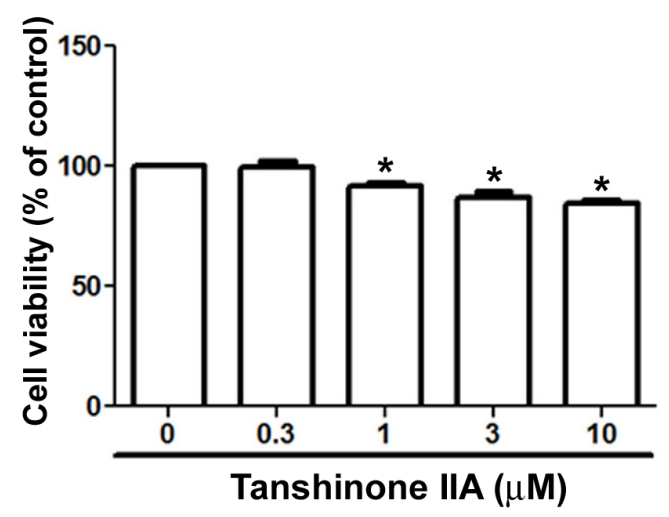

B

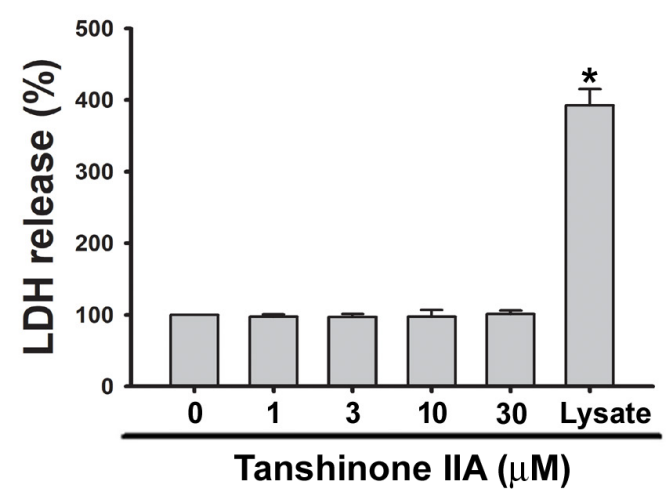

Figure 1: Effects of tanshinone IIA on cell growth and cytotoxicity in human EPCs. EPCs were incubated with the indicated concentrations of tanshinone IIA for $24 \mathrm{~h}$, then cell viability (A) and cytotoxicity (B) were determined using the MTT and LDH assays, respectively. Data represent the mean \pm S.E.M. of four independent experiments. ${ }^{*}, p<0.05$ compared with the control group.

A

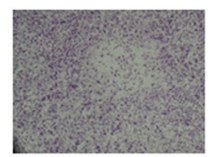

control

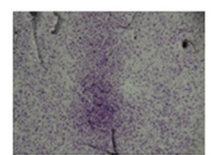

0
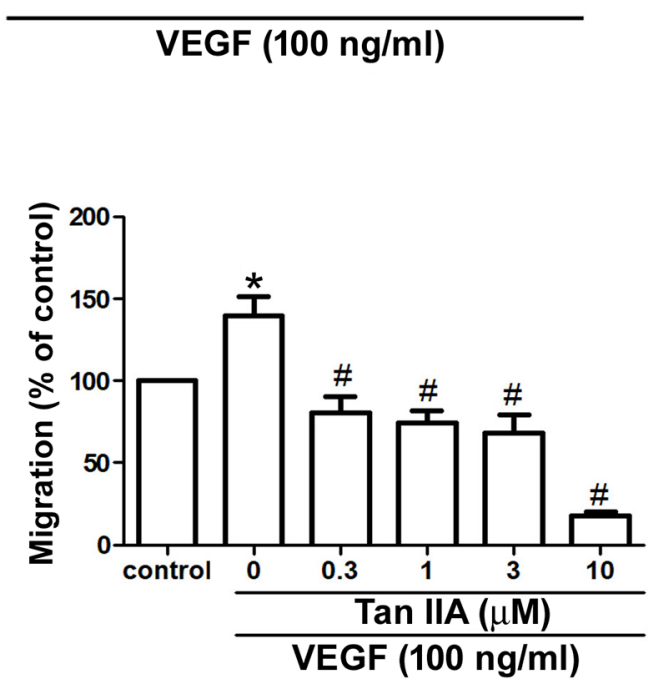

B
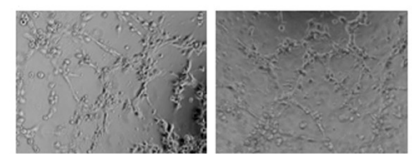

0.3

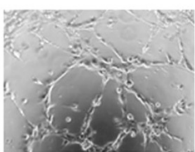

control
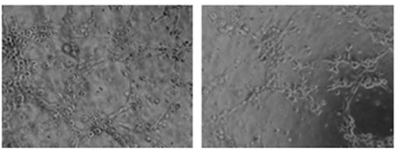

3

1 Tan IIA $(\mu \mathrm{M})$

0

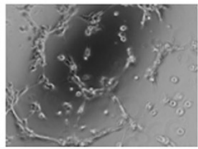

10 Tan IIA $(\mu \mathrm{M})$

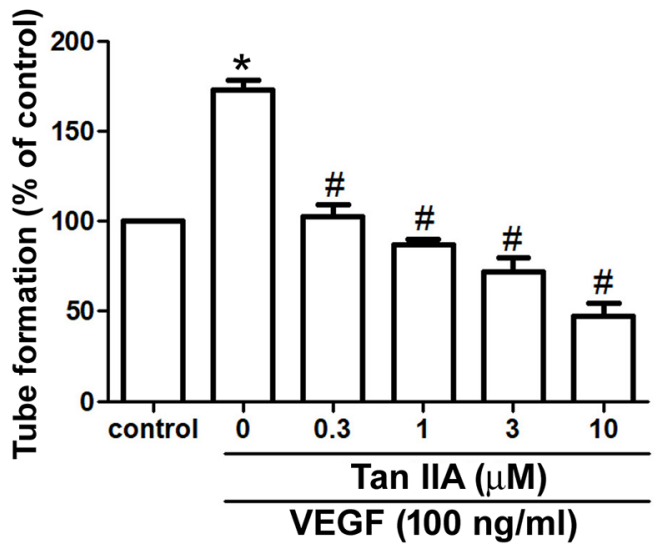

Figure 2: Effects of tanshinone IIA on VEGF-induced migration and tube formation in human EPCs. EPCs were stimulated with or without VEGF $(100 \mathrm{ng} / \mathrm{ml})$ in the absence or presence of various concentrations of tanshinone IIA for $24 \mathrm{~h}$. Cell migration (A) and capillary-like structure formation (B) were examined by Transwell and tube formation assays, respectively. Data represent the mean \pm S.E.M. of four independent experiments. ${ }^{*}, p<0.05$ compared with the control group; ${ }^{*}, p<0.05$ compared with the VEGF-treated group. 
that noncytotoxic concentrations of tanshinone IIA exhibit promising anti-angiogenic effects in human EPCs.

\section{Tanshinone IIA inhibits VEGF-induced PLC and Akt activation in EPCs}

PLC and Akt activation are required to facilitate angiogenesis in EPCs [31, 32]. We therefore examined whether the inhibitory effect of tanshinone IIA is due to its ability to interfere with VEGF-induced activation of the PLC and Akt pathways. Incubation of EPCs with VEGF promoted the phosphorylation of PLC and Akt, whereas VEGF-induced PLC and Akt phosphorylation was inhibited by tanshinone IIA treatment in a concentration-dependent manner (Figure 3 ). Thus, we suggest that tanshinone IIA may suppress VEGF-induced EPC angiogenesis via the PLC- and Akt-dependent pathways. Evidence indicates the involvement of the PDK1, PI3K and mTOR signaling pathways in Akt activation [33]. However, we found that tanshinone IIA did not affect PDK1, PI3K, or mTOR phosphorylation (Supplementary Figure 2). Whether there is some other signaling interaction with Akt needs to be investigated.

\section{Tanshinone IIA inhibits VEGF-induced JNK but not ERK and p38 phosphorylation in EPCs}

The mitogen-activated protein kinase (MAPK) pathways regulate various biological functions of
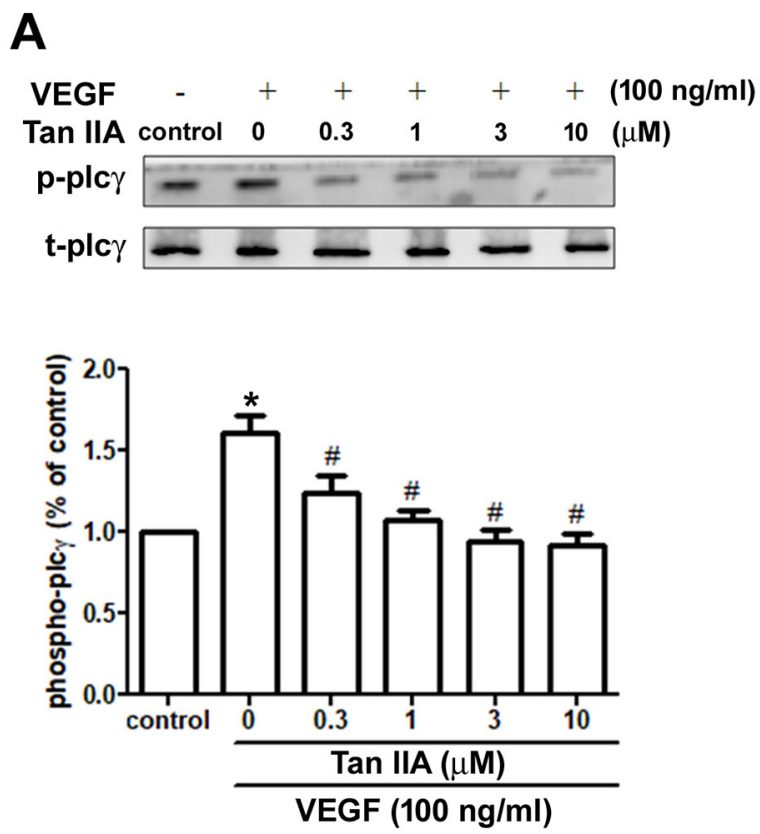

endothelial cells for angiogenesis [34, 35]. We evaluated whether the JNK, ERK and 38 protein kinases are involved in the anti-angiogenic effect of tanshinone IIA. As shown in Figure 4, stimulation of EPCs with VEGF significantly increased JNK phosphorylation, but not ERK and p38 phosphorylation. Tanshinone IIA dramatically suppressed VEGF-induced JNK activation in a concentrationdependent manner. These results demonstrate that the JNK signaling pathway is involved in tanshinone IIA-induced anti-angiogenic activity in human EPCs.

\section{Tanshinone IIA inhibits angiogenesis and the expression of progenitor cell marker in vivo}

We performed the in vivo CAM assay to characterize the effect of tanshinone IIA on angiogenesis. Whereas VEGF increased vessel formation in the CAM model, tanshinone IIA significantly repressed VEGF-enhanced vessel formation in a dose-dependent manner (Figure 5). The Matrigel implant assay in mice was used to confirm the in vivo anti-angiogenic activity of tanshinone IIA. We found that VEGF promoted microvessel formation and hemoglobin content in Matrigel plugs, whereas tanshinone IIA profoundly inhibited this process (Figure 6A and 6B). Immunohistochemical staining demonstrated the presence of the vessel marker CD31 and EPC-specific markers CD34 and CD133, all of which were visibly reduced by tanshinone IIA (Figure 6C). Taken together, these data reveal that tanshinone IIA impedes EPC-regulated angiogenesis in vivo.

B
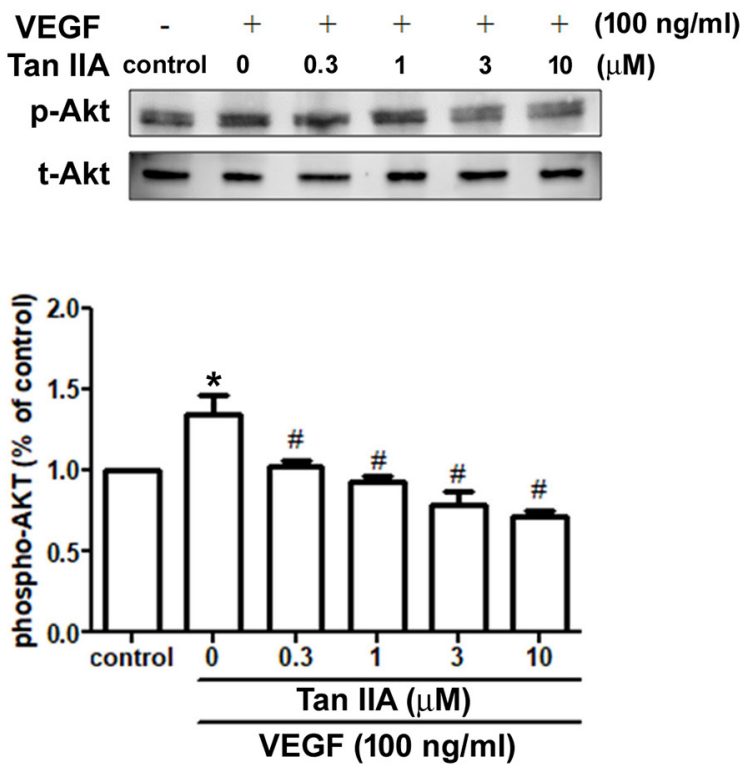

Figure 3: Effects of tanshinone IIA on PLC and Akt signaling pathways in human EPCs. EPCs were incubated with VEGF-A (100 ng/ml) and the indicated concentrations of tanshinone IIA for $24 \mathrm{~h}$. Phosphorylation of PLC (A) and Akt (B) was examined by Western blotting. Data represent the mean \pm S.E.M. of four independent experiments. ${ }^{*}, p<0.05$ compared with the control group; ${ }^{*}, p$ $<0.05$ compared with the VEGF-treated group. 
A
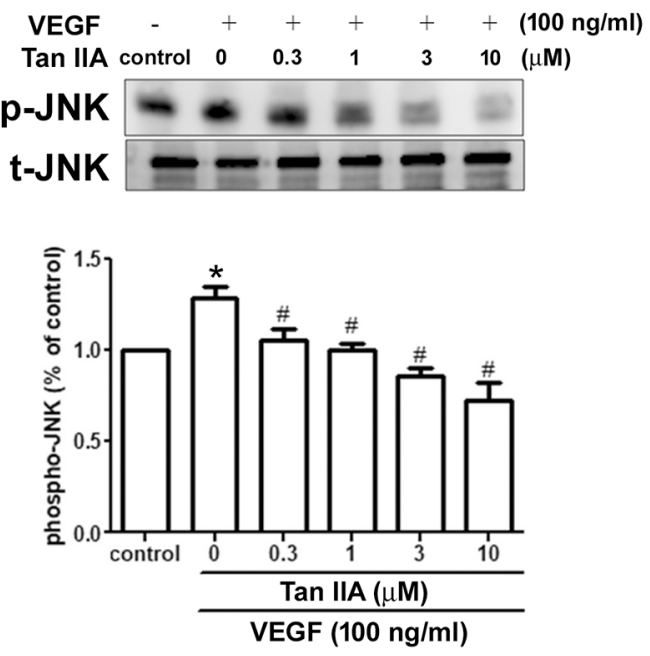

B
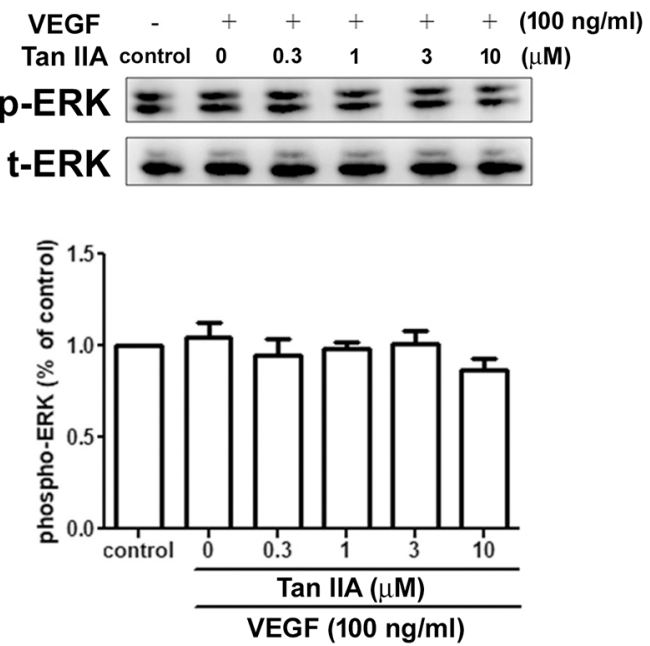

C
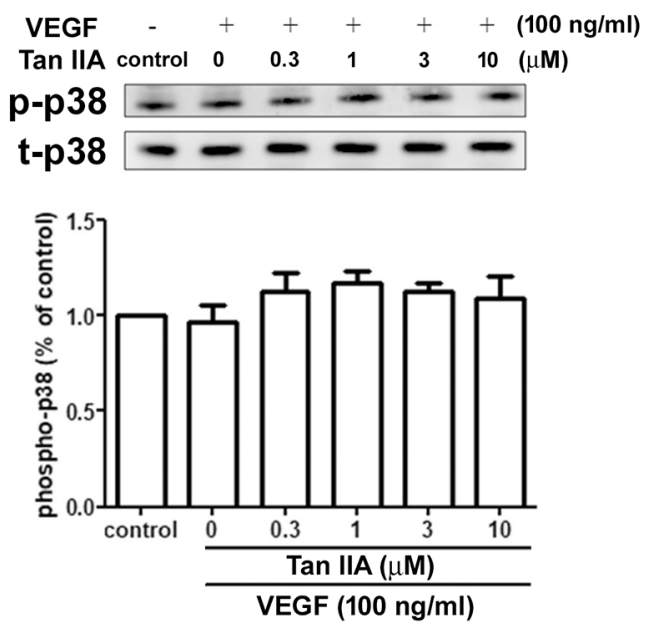

Figure 4: Effects of tanshinone IIA on the MAPK pathway in human EPCs. EPCs were incubated with VEGF-A (100 ng/ml) and the indicated concentrations of tanshinone IIA for $24 \mathrm{~h}$. Phosphorylation of JNK (A), ERK (B) and p38 (C) was examined by Western blotting. Data represent the mean \pm S.E.M. of four independent experiments. ${ }^{*}, p<0.05$ compared with the control group; ${ }^{\#}, p<0.05$ compared with the VEGF-treated group. 


\section{DISCUSSION}

The metastatic cascade is a multi-step process encompassing local invasion of tumor cells into the adjacent tissue [23], transendothelial migration of cancer cells into vessels [36], angiogenesis [37] and proliferation [38]. Angiogenesis is the most critical step, as the growing tumor requires a supply of oxygen and nutrients to ensure its growth. The metastatic spread of tumors also depends on growth of the vascular network. Angiogenesis inhibitors represent a breakthrough in the treatment of cancer and age-related macular degeneration (AMD). [39]. Compelling evidence indicates that tumor angiogenesis is supported by the mobilization and functional incorporation of other cells such as EPCs [40]. A recent study demonstrates that EPCs promote early tumor growth and also late tumor metastasis by activating the angiogenic switch. In addition, EPCs contribute to neovessel formation in tumors by secreting abundant angiogenic growth factors during tumor development [41]. It has been reported that certain chemotherapy drugs can trigger the mobilization of circulating EPCs and their subsequent 'homing' into the tumor [42]. EPC-targeting therapies may therefore be a promising strategy to block angiogenesis-facilitated tumor growth and metastasis. In this study, we used EPCs to investigate the antiangiogenic effects of tanshinone IIA. Our results showed that tanshinone IIA inhibited VEGF-induced migration and tube formation of human EPCs in a concentrationdependent manner. We also provide evidence showing that the anti-angiogenic effects of tanshinone IIA in EPCs are not related to cytotoxicity. Importantly, tanshinone IIA markedly suppressed in vivo angiogenesis and the expression of specific EPC markers in an animal tumor model. Based upon these findings, we suggest that tanshinone IIA may be a potential angiogenesis inhibitor that targets EPCs.

Salvia miltiorrhiza (Danshen) is a TCM product with a long history of good efficacy and tolerability in the treatment of cardiovascular diseases. Danshen and its preparations have been reported to be effective treatments for congestive heart failure and angina pectoris $[16,43]$. Tanshinone IIA is the major active compound in Danshen, with documented cardiovascular bioactivity and safety

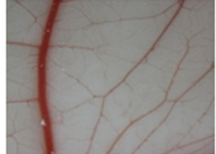

control

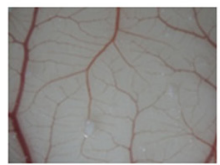

0

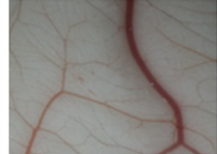

0.3

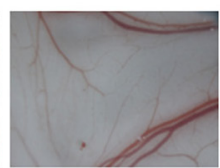

3

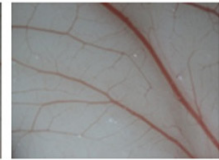

1 Tan IIA $(\mu \mathrm{M})$

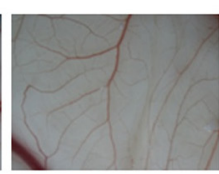

10 Tan IIA $(\mu \mathrm{M})$

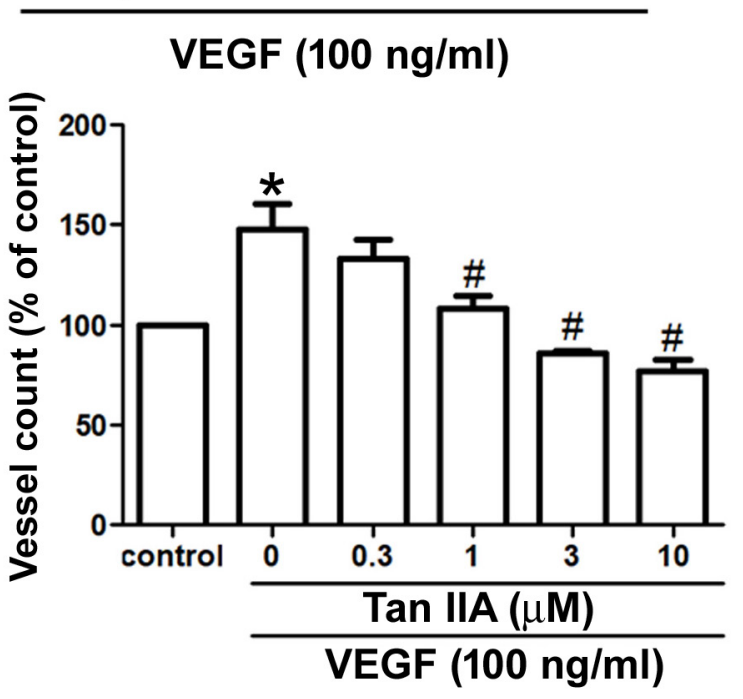

Figure 5: Effects of tanshinone IIA on VEGF-induced angiogenesis in the CAM model. Five-day-old fertilized chick embryos were treated with or without VEGF $(100 \mathrm{ng} / \mathrm{ml})$ in the absence or presence of various concentrations of tanshinone IIA for 3 days. After treatment, the CAMs were examined by microscopy and photographed. Data represent the mean \pm S.E.M. ( $\mathrm{N}$ number $\geq 8$ chick embryos per group). ${ }^{*}, p<0.05$ compared with the control group; ${ }^{\#}, p<0.05$ compared with the VEGF-treated group. 


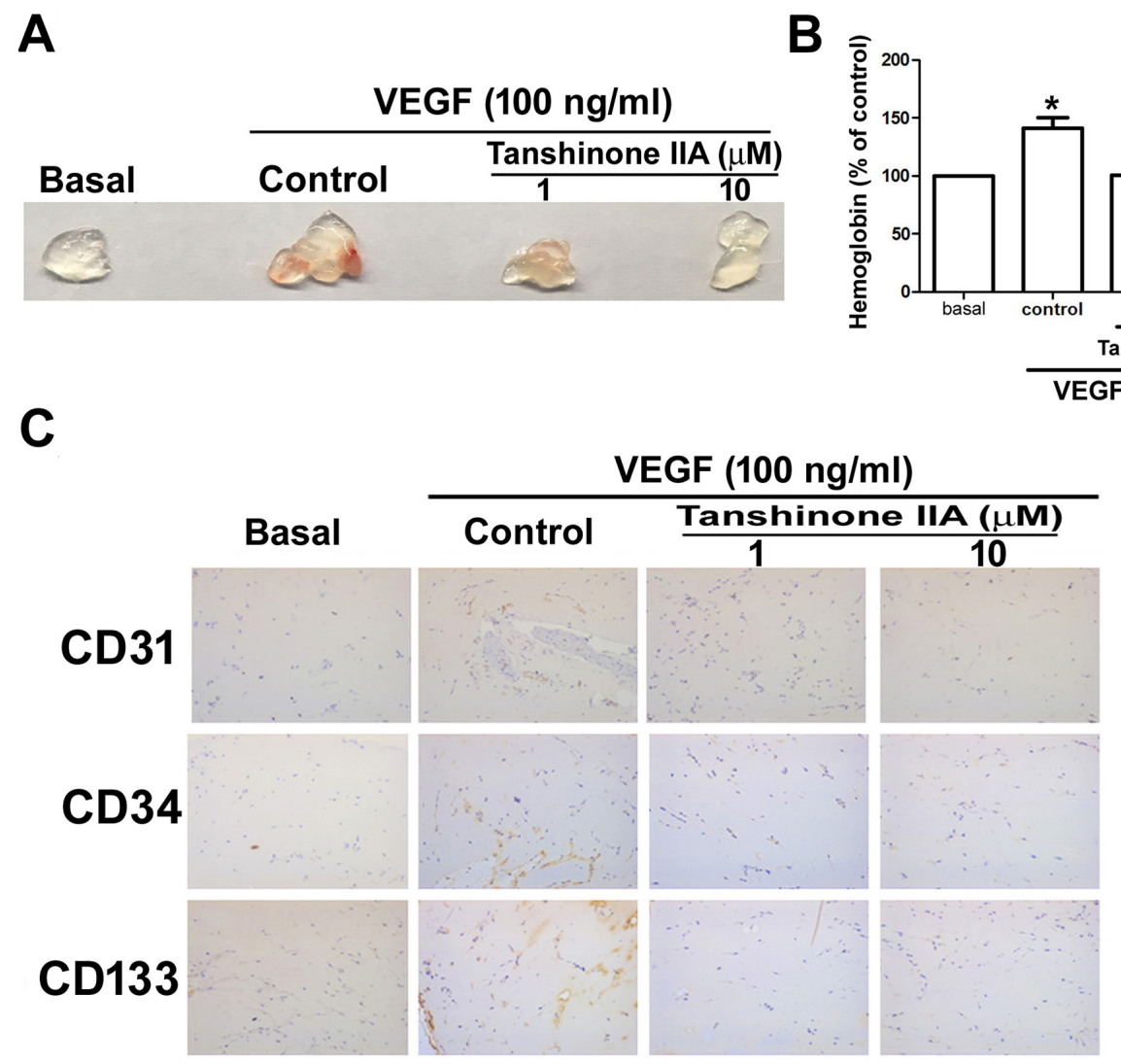

Figure 6: Effects of tanshinone IIA on in vivo angiogenesis and EPC marker expression in the Matrigel plug model. Matrigel plugs were treated with PBS (basal group) or VEGF (control group) in the absence or presence of tanshinone IIA (1 and $10 \mu \mathrm{M})$ and subcutaneously injected into the flanks of nude mice (N number $\geq 8$ mice per group). After 7 days, the plugs were photographed (A) and hemoglobin levels were quantified (B). Specimens from the plugs were immunostained with antibodies against CD31, CD34 and CD133 (C). Data represent the mean \pm S.E.M. of at least 5 mice per group. ${ }^{*}, p<0.05$ compared with the basal group; ${ }^{*}, p<0.05$ compared with the control group.

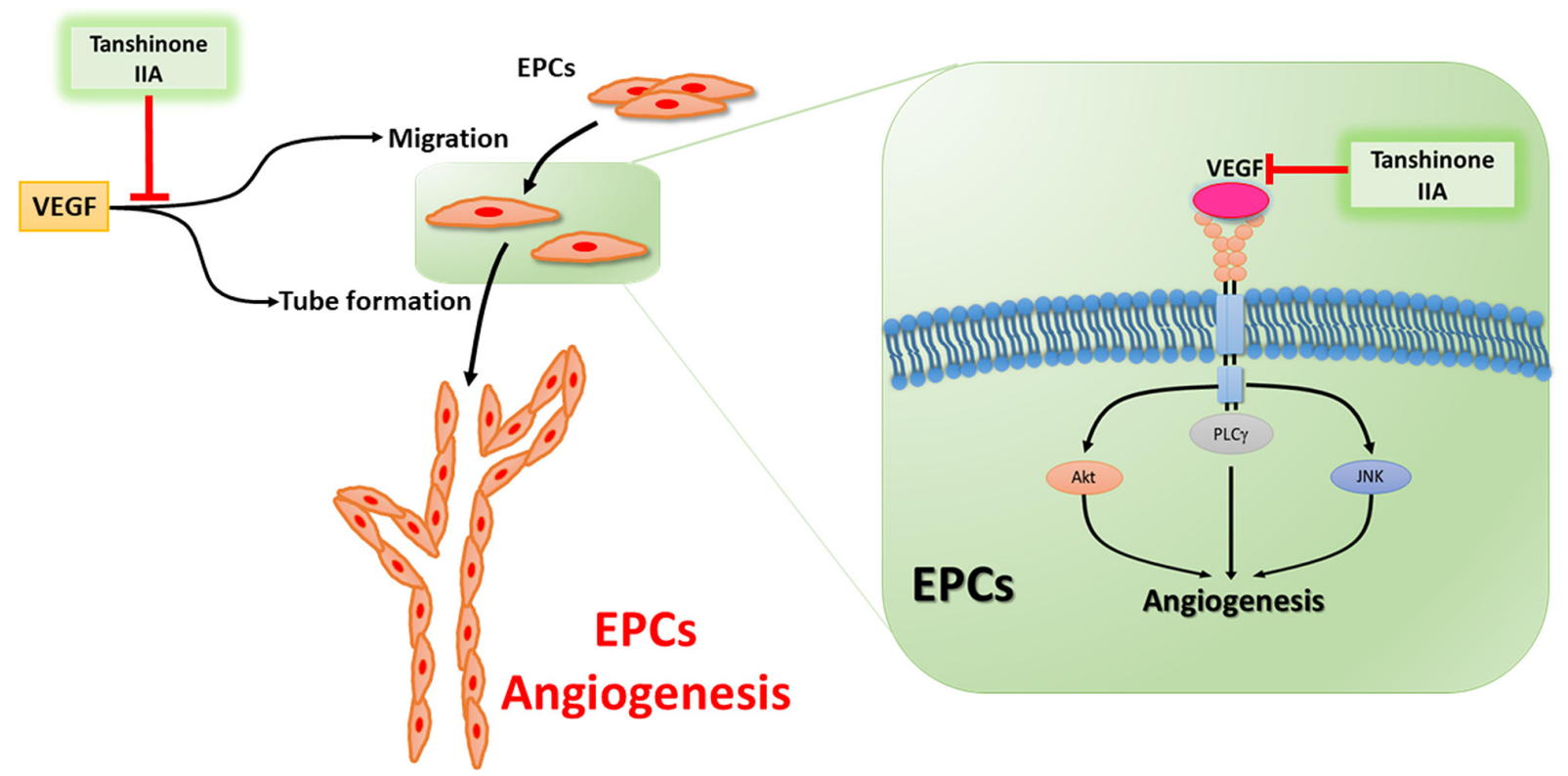

Figure 7: Schema of tanshinone IIA-induced anti-angiogenesis property in human endothelial progenitor cells. Tanshinone IIA reduces EPCs angiogenesis in vitro and in vivo by controlling the PLC, Akt and JNK signaling pathways. 
[44]. Previous studies have shown that tanshinone IIA exhibits anti-angiogenic activity in HUVECs [21, 22]. In this study, we report for the first time that tanshinone IIA anti-angiogenic effects in human EPCs. Our data reveal that nontoxic concentrations of tanshinone IIA inhibit EPC angiogenesis in vitro and in vivo. Tanshinone IIA is a promising natural product worthy of further investigation for the treatment of angiogenesis-related diseases. Tanshinone IIA is the main constituent in Bushen Huoxue Qubi (BHQ) granules, a TCM preparation that is used clinically for the treatment of blood stasis syndrome. A pharmacokinetics and tissue distribution study involving rats with acute blood stasis treated with orally administered BHQ has reported significantly higher values for the area under the concentration-time curve (AUC), maximum plasma concentration $\left(\mathrm{C}_{\max }\right)$, biological halflife $\left(t_{1 / 2}\right)$, lower total body clearance $(C L)$ and apparent volume of distribution (Vd) of tanshinone IIA in plasma and higher $\mathrm{AUC}_{0-\mathrm{t}}$ of tanshinone IIA in the analyzed tissues of the BHQ-treated rats, compared with normal rats [45]. In addition, high density lipoprotein (HDL)-loaded tanshinone IIA markedly improved pharmacokinetic behaviors of tanshinone IIA in vivo [46]. Whether HDLtanshinone IIA has greater anti-angiogenic effects than tanshinone IIA in EPCs needs further examination. Evidence indicates that tanshinone IIA $(20-100 \mu \mathrm{M})$ induces osteosarcoma apoptosis [47]. This current study suggests that tanshinone IIA at low concentrations (0.3 $10 \mu \mathrm{M}$ ) reduces EPC-associated angiogenesis, indicating that the effective dose of tanshinone IIA in EPCs has physiologically relevant antitumor activity.

The MAPK pathway plays a key role in VEGFregulated angiogenesis [34]. Here, we found that VEGF only significantly promoted the phosphorylation of JNK, and not ERK and p38, in EPCs. Notably, tanshinone IIA concentration-dependently diminished VEGF-induced JNK phosphorylation, indicating that tanshinone IIA may suppress VEGF-dependent EPC angiogenesis through the JNK signaling pathway. Involvement of the PLC pathway has been documented in VEGF-dependent angiogenesis [31]. Akt signaling is also a critical mediator in EPC angiogenesis [32]. In this study, VEGF-induced PLC and Akt phosphorylation was dramatically reversed by tanshinone IIA in EPCs. These results suggest that the PLC and Akt pathways are probably both involved in tanshinone IIA-induced suppression of EPC angiogenesis. Whether any crosstalk exists between the PLC, Akt and JNK pathways after tanshinone IIA treatment deserves further investigation.

In conclusion, the current report discloses a novel mechanism by which tanshinone IIA reduces EPC angiogenesis in vitro and in vivo. We demonstrate that tanshinone IIA antagonizes EPC migration and tube formation by controlling the PLC, Akt and JNK signaling pathways (Figure 7). EPCs have been characterized as having the ability to dictate angiogenesis and metastasis in the tumor microenvironment. To our knowledge, this study is the first to identify that tanshinone IIA has anti-angiogenic activity against human EPCs. Our findings suggest that tanshinone IIA may serve as a lead candidate for further development of novel angiogenic inhibitors that are capable of blocking cancer progression and metastasis.

\section{MATERIALS AND METHODS}

\section{Materials}

Anti-CD133 antibody (Cat. No. orb13002) was obtained from Biorbyt (Biorbyt, Cambridge, UK). CD31 (Cat. No. ab9498) and CD34 (Cat. No. ab81289) antibodies were obtained from Abcam (Cambridge, MA, USA). Recombinant human VEGF was bought from PeproTech (Rocky Hill, NJ, USA). Anti-p-PLC (SC-136186), anti-p-Akt (SC-16646-R), anti-Akt (SC5298), anti-p-ERK (SC-7383), anti-ERK (SC-154), anti-p-p38 (SC-166182), anti-p38 (SC-535), anti-p-JNK (SC-6254), anti-JNK (SC-7345), anti-mouse and antirabbit conjugated horseradish peroxidase antibodies were purchased from Santa Cruz Biotechnology (Santa Cruz, CA, USA).

\section{Cell culture}

The study protocol for isolation of human EPCs was approved by the Institutional Review Board of Mackay Memorial Hospital, Taipei, Taiwan (reference number: 13MMHIS062). Peripheral blood mononuclear cells (PBMCs) were centrifuged from healthy donors using Ficoll-Paque PLUS solution (Amersham Biosciences, Uppala, Sweden), according to the manufacturer's protocol. $\mathrm{CD}^{+} 4^{+}$progenitor cells were isolated from PBMCs using the CD34 MicroBead kit and MACS Cell Separation System (Miltenyi Biotec, Bergisch Gladbach, Germany), then cultured in MV2 complete medium containing MV2 basal medium and growth supplement (PromoCell, Heidelberg, Germany) supplemented with 20\% defined fetal bovine serum (FBS) (HyClone, Logan, UT, USA) and maintained at $37^{\circ} \mathrm{C}$ in a humidified atmosphere of $5 \% \mathrm{CO}_{2}[23,24]$.

\section{Cell viability assay}

EPCs were treated with or without tanshinone IIA for $24 \mathrm{~h}$, then subjected to 3-(4,5-dimethylthiazol-2-yl)2,5-diphenyltetrazolium bromide (MTT; $0.5 \mathrm{mg} / \mathrm{mL}$ ) for $30 \mathrm{~min}$. MTT was dissolved in dimethylsulfoxide (DMSO) and the absorbance was measured at $550 \mathrm{~nm}$ using a microplate reader (Bio-Tek, Winooski, VT, USA) [25].

\section{Cytotoxicity assay}

EPCs were seeded onto 96-well plates in a density of $5 \times 10^{3}$ cells per well. After $24 \mathrm{~h}$ incubation, cells 
were treated with MV2 complete medium containing 2\% FBS in the absence or presence of tanshinone IIA. The percentage of LDH release in the collected medium was determined using a non-radioactive cytotoxicity assay kit (Promega, Madison, WI, USA).

\section{Western blot analysis}

EPCs were lysed and processed by sodium dodecyl sulfate-polyacrylamide gel electrophoresis and transferred to Immobilon polyvinyldifluoride membranes. The blots were blocked with $4 \%$ bovine serum albumin then probed with primary antibodies, before being treated with donkey anti-rabbit peroxidase-conjugated secondary antibody. Finally, the blots were visualized by enhanced chemiluminescence using an ImageQuant LAS 4000 system (GE Healthcare, Pewaukee, WI, USA) [26].

\section{Measurement of EPC migratory ability}

EPCs $\left(5 \times 10^{4}\right.$ cells/well) were seeded onto the upper chamber with MV2 complete medium, then incubated in the bottom chamber with MV2 complete medium containing VEGF with the indicated concentrations of tanshinone IIA. After $24 \mathrm{~h}$ of treatment, cells on the upper side of the filters were mechanically removed, and those that had migrated to the lower side were fixed with $4 \%$ formaldehyde, then stained with $0.05 \%$ crystal violet. Cells on the upper side of the Transwell membrane were photographed and counted under a microscope [27].

\section{Measurement of EPC tube formation}

EPCs $\left(3 \times 10^{4}\right.$ cells $)$ were seeded onto pre-coated Matrigel plates (BD Biosciences, Bedford, MA, USA) in MV2 complete medium containing VEGF with the indicated concentrations of tanshinone IIA, followed by incubation for $24 \mathrm{~h}$ at $37^{\circ} \mathrm{C}$. EPC tube formation was photographed and numbers of tube branches were calculated using MacBiophotonics Image J software [27, 28].

\section{Chick chorioallantoic membrane (CAM) assay}

VEGF and the indicated concentrations of tanshinone IIA were mixed with Matrigel and applied into the center of the developing chorioallantoic membrane of a fertilized chicken egg. After 3 days, CAMs were collected for microscopy and photographic documentation. Angiogenesis was quantified by counting the number of blood vessel branches; at least 10 viable embryos were tested for each treatment.

\section{Matrigel plug assay}

Four-week-old nude male mice were subcutaneously injected with $300 \mu \mathrm{l}$ Matrigel containing VEGF with the indicated concentrations of tanshinone IIA. The plugs were collected after 7 days and then fixed, embedded in paraffin, and processed by immunohistochemical staining for CD31, CD34 and CD133 antibodies [29]. Hemoglobin content was examined using Drabkin's reagent. All animal procedures were performed according to approved protocols issued by the China Medical University (Taichung, Taiwan) Institutional Animal Care and Use Committees.

\section{Statistical analysis}

Data are presented as the mean \pm standard error of the mean. Statistical analysis of comparisons between 2 samples was performed using the Student's $t$ test. Statistical comparisons of more than 2 groups were performed using one-way analysis of variance with Bonferroni's post-hoc test. In all cases, $p<0.05$ was considered statistically significant.

\section{ACKNOWLEDGMENTS AND FUNDING}

This work was supported by grants from the Ministry of Science and Technology of Taiwan (MOST-102-2632B-715-001-MY3; MOST-103-2320-B-075A-001-MY3; MOST-103-2628-B-039-002; MOST 105-2632-B-715001; MOST 106-2320-B-715 -001-MY3); Mackay Medical College (MMC-1051B13) and China Medical University Beigang Hospital (CMUBH R105-006).

\section{CONFLICTS OF INTEREST}

None of the authors of this paper has any financial or personal relationships with other people or organizations that could inappropriately influence this work.

\section{REFERENCES}

1. Folkman J. Angiogenesis. Annual review of medicine. 2006; 57:1-18.

2. Carmeliet P, Jain RK. Angiogenesis in cancer and other diseases. Nature. 2000; 407:249-257.

3. Jain RK. Antiangiogenesis strategies revisited: From starving tumors to alleviating hypoxia. Cancer cell. 2014; 26:605-622.

4. Lii CK, Chang JW, Chen JJ, Chen HW, Liu KL, Yeh SL, Wang TS, Liu SH, Tsai CH, Li CC. Docosahexaenoic acid inhibits 12-O-tetradecanoylphorbol-13- acetateinduced fascin-1-dependent breast cancer cell migration by suppressing the pkcdelta- and wnt-1/beta-catenin-mediated pathways. Oncotarget. 2016; 7:25162-25179. http://doi. org/10.18632/oncotarget.7301.

5. Su CM, Huang CY, Tang CH. Characteristics of resistin in rheumatoid arthritis angiogenesis. Biomarkers in medicine. 2016; 10:651-660.

6. Chung AS, Lee J, Ferrara N. Targeting the tumour vasculature: Insights from physiological angiogenesis. Nature reviews Cancer. 2010; 10:505-514. 
7. Simone V, Brunetti O, Lupo L, Testini M, Maiorano E, Simone M, Longo V, Rolfo C, Peeters M, Scarpa A, Azzariti A, Russo A, Ribatti D, Silvestris N. Targeting angiogenesis in biliary tract cancers: An open option. International journal of molecular sciences. 2017; 18.

8. Petrovic N. Targeting Angiogenesis in Cancer Treatments: Where do we Stand? Journal of pharmacy \& pharmaceutical sciences : a publication of the Canadian Society for Pharmaceutical Sciences, Societe canadienne des sciences pharmaceutiques. 2016; 19:226-238.

9. Asahara T, Masuda H, Takahashi T, Kalka C, Pastore C, Silver M, Kearne M, Magner M, Isner JM. Bone marrow origin of endothelial progenitor cells responsible for postnatal vasculogenesis in physiological and pathological neovascularization. Circulation research. 1999; 85:221-228.

10. Yoder MC. Human endothelial progenitor cells. Cold Spring Harbor perspectives in medicine. 2012; 2:a006692.

11. Peters BA, Diaz LA, Polyak K, Meszler L, Romans K, Guinan EC, Antin JH, Myerson D, Hamilton SR, Vogelstein B, Kinzler KW, Lengauer C. Contribution of bone marrowderived endothelial cells to human tumor vasculature. Nature medicine. 2005; 11:261-262.

12. Jain RK, Carmeliet P. Snapshot: Tumor angiogenesis. Cell. 2012; 149:1408-1408 e1401.

13. Miao ZH, Feng JM, Ding J. Newly discovered angiogenesis inhibitors and their mechanisms of action. Acta pharmacologica Sinica. 2012; 33:1103-1111.

14. Wang YQ, Miao ZH. Marine-derived angiogenesis inhibitors for cancer therapy. Marine drugs. 2013; 11:903-933.

15. Su CM, Wang SW, Lee TH, Tzeng WP, Hsiao CJ, Liu SC, Tang CH. Trichodermin induces cell apoptosis through mitochondrial dysfunction and endoplasmic reticulum stress in human chondrosarcoma cells. Toxicology and applied pharmacology. 2013; 272:335-344.

16. Wu WY, Wang YP. Pharmacological actions and therapeutic applications of salvia miltiorrhiza depside salt and its active components. Acta pharmacologica Sinica. 2012; 33:1119-1130.

17. Padma VV. An overview of targeted cancer therapy. BioMedicine. 2015; 5:19.

18. Zhang Y, Jiang $\mathrm{P}$, Ye M, Kim SH, Jiang C, Lu J. Tanshinones: Sources, pharmacokinetics and anti-cancer activities. International journal of molecular sciences. 2012; 13:13621-13666.

19. Fan GW, Gao XM, Wang H, Zhu Y, Zhang J, Hu LM, Su YF, Kang LY, Zhang BL. The anti-inflammatory activities of tanshinone IIA, an active component of tcm, are mediated by estrogen receptor activation and inhibition of inos. The Journal of steroid biochemistry and molecular biology. 2009; 113:275-280.

20. Chang CC, Chu CF, Wang CN, Wu HT, Bi KW, Pang JH, Huang ST. The anti-atherosclerotic effect of tanshinone IIA is associated with the inhibition of TNF-alpha-induced
VCAM-1, ICAM-1 and CX3CL1 expression. Phytomedicine : international journal of phytotherapy and phytopharmacology. 2014; 21:207-216.

21. Wang Y, Li JX, Wang YQ, Miao ZH. Tanshinone i inhibits tumor angiogenesis by reducing Stat3 phosphorylation at Tyr705 and hypoxia-induced HIF-1alpha accumulation in both endothelial and tumor cells. Oncotarget. 2015; 6:16031-16042. http://doi.org/10.18632/oncotarget.3648.

22. Xing Y, Tu J, Zheng L, Guo L, Xi T. Anti-angiogenic effect of tanshinone IIA involves inhibition of the VEGF/ VEGFR2 pathway in vascular endothelial cells. Oncology reports. 2015; 33:163-170.

23. Wu MH, Huang CY, Lin JA, Wang SW, Peng CY, Cheng $\mathrm{HC}$, Tang $\mathrm{CH}$. Endothelin-1 promotes vascular endothelial growth factor-dependent angiogenesis in human chondrosarcoma cells. Oncogene. 2014; 33:1725-1735.

24. Yu HS, Wang SW, Chang AC, Tai HC, Yeh HI, Lin YM, Tang $\mathrm{CH}$. Bradykinin promotes vascular endothelial growth factor expression and increases angiogenesis in human prostate cancer cells. Biochem Pharmacol. 2014; 87:243-253.

25. Liu JF, Chen CY, Chen HT, Chang CS, Tang CH. B1-038, a benzofuran derivative, induces cell apoptosis in human chondrosarcoma cells through reactive oxygen species/ mitochondrial dysfunction and the caspases dependent pathway. International journal of molecular sciences. 2016; 17.

26. Huang CY, Chen SY, Tsai HC, Hsu HC, Tang CH. Thrombin induces epidermal growth factor receptor transactivation and CCL2 expression in human osteoblasts. Arthritis and rheumatism. 2012; 64:3344-3354.

27. Su CM, Hsu CJ, Tsai CH, Huang CY, Wang SW, Tang CH. Resistin promotes angiogenesis in endothelial progenitor cells through inhibition of microRNA206: Potential implications for rheumatoid arthritis. Stem cells. 2015; 33:2243-2255.

28. Chen CY, Su CM, Hsu CJ, Huang CC, Wang SW, Liu $\mathrm{SC}$, Chen WC, Fuh LJ, Tang CH. CCN1 promotes VEGF production in osteoblasts and induces endothelial progenitor cell angiogenesis by inhibiting miR-126 expression in rheumatoid arthritis. Journal of bone and mineral research : the official journal of the American Society for Bone and Mineral Research. 2017; 32:34-45.

29. Passaniti A, Taylor RM, Pili R, Guo Y, Long PV, Haney JA, Pauly RR, Grant DS, Martin GR. A simple, quantitative method for assessing angiogenesis and antiangiogenic agents using reconstituted basement membrane, heparin, and fibroblast growth factor. Lab Invest. 1992; 67:519-528.

30. Ammendola $M$, Leporini C, Luposella $M$, Sacco $R$, Sammarco G, Russo E, Patruno R, De Sarro G, Ranieri G. Targeting endothelial progenitor cells in cancer as a novel biomarker and anti-angiogenic therapy. Current stem cell research \& therapy. 2015; 10:181-187. 
31. Wang LH, Tsai HC, Cheng YC, Lin CY, Huang YL, Tsai $\mathrm{CH}, \mathrm{Xu} \mathrm{GH}$, Wang SW, Fong YC, Tang CH. CTGF promotes osteosarcoma angiogenesis by regulating miR-543/ angiopoietin 2 signaling. Cancer letters. 2017; 391:28-37.

32. Li TM, Liu SC, Huang YH, Huang CC, Hsu CJ, Tsai $\mathrm{CH}$, Wang SW, Tang CH. Ykl-40-induced inhibition of miR-590-3p promotes interleukin-18 expression and angiogenesis of endothelial progenitor cells. International journal of molecular sciences. 2017; 18.

33. Bertacchini J, Heidari N, Mediani L, Capitani S, Shahjahani M, Ahmadzadeh A, Saki N. Targeting PI3K/AKT/mTOR network for treatment of leukemia. Cellular and molecular life sciences : CMLS. 2015; 72:2337-2347.

34. Liao YY, Tsai HC, Chou PY, Wang SW, Chen HT, Lin YM, Chiang IP, Chang TM, Hsu SK, Chou MC, Tang CH, Fong YC. CCL3 promotes angiogenesis by dysregulation of miR-374b/VEGF-a axis in human osteosarcoma cells. Oncotarget. 2016; 7:4310-4325. http://doi.org/10.18632/ oncotarget.6708.

35. Varinska L, Gal P, Mojzisova G, Mirossay L, Mojzis J. Soy and breast cancer: Focus on angiogenesis. International journal of molecular sciences. 2015; 16:11728-11749.

36. Hsieh JY, Huang TS, Cheng SM, Lin WS, Tsai TN, Lee OK, Wang HW. miR-VEGFs146a-5p circuitry uncouples cell proliferation and migration, but not differentiation, in human mesenchymal stem cells. Nucleic acids research. 2013.

37. Bhat TA, Nambiar D, Tailor D, Pal A, Agarwal R, Singh RP. Acacetin inhibits in vitro and in vivo angiogenesis and down-regulates stat signaling and VEGF expression. Cancer Prev Res (Phila). 2013.

38. Ling N, Gu J, Lei Z, Li M, Zhao J, Zhang HT, Li X. Microrna-155 regulates cell proliferation and invasion by targeting FOXO3a in glioma. Oncology reports. 2013.

39. Cook KM, Figg WD. Angiogenesis inhibitors: Current strategies and future prospects. CA: a cancer journal for clinicians. 2010; 60:222-243.
40. Urbich C, Dimmeler S. Endothelial progenitor cells: Characterization and role in vascular biology. Circulation research. 2004; 95:343-353.

41. Seandel M, Butler J, Lyden D, Rafii S. A catalytic role for proangiogenic marrow-derived cells in tumor neovascularization. Cancer cell. 2008; 13:181-183.

42. Shaked Y, Ciarrocchi A, Franco M, Lee CR, Man S, Cheung AM, Hicklin DJ, Chaplin D, Foster FS, Benezra R, Kerbel RS. Therapy-induced acute recruitment of circulating endothelial progenitor cells to tumors. Science. 2006; 313:1785-1787.

43. Chen YJ, Chung TY, Chen WY, Chen CY, Lee MR, Jinn TR, Tc Tzen J. Detecting metabolites of different transition metal-lithospermate B complexes after intravenous injection in rats. Acta pharmacologica Sinica. 2014; 35:937-944.

44. Dong Y, Morris-Natschke SL, Lee KH. Biosynthesis, total syntheses, and antitumor activity of tanshinones and their analogs as potential therapeutic agents. Natural product reports. 2011; 28:529-542.

45. Wang Y, Yan J, Li S, Cai X, Wang W, Luo K, Huang D, Gao J. Pharmacokinetics and tissue distribution study of tanshinone IIA after oral administration of bushen huoxue qubi granules to rats with blood stasis syndrome. Pharmacognosy magazine. 2014; 10:285-291.

46. Zhang W, He H, Liu J, Wang J, Zhang S, Zhang S, Wu Z. Pharmacokinetics and atherosclerotic lesions targeting effects of tanshinone IIA discoidal and spherical biomimetic high density lipoproteins. Biomaterials. 2013; 34:306-319.

47. Huang ST, Huang CC, Huang WL, Lin TK, Liao PL, Wang PW, Liou CW, Chuang JH. Tanshinone IIA induces intrinsic apoptosis in osteosarcoma cells both in vivo and in vitro associated with mitochondrial dysfunction. Scientific reports. 2017; 7:40382. 